\title{
Adiponectin and its receptors are involved in hypertensive vascular injury
}

\author{
RUIMIN GUO, MIN HAN, JUAN SONG, JUN LIU and YANNI SUN \\ Emergency Medicine, Putuo Hospital Affiliated to Shanghai Traditional Chinese Medicine University, \\ Shanghai 200333, P.R. China
}

Received November 29, 2016; Accepted September 7, 2017

DOI: $10.3892 / \mathrm{mmr} .2017 .7878$

\begin{abstract}
Adipocyte-derived adiponectin (APN) is involved in the protection against cardiovascular disease, but the endogenous APN and its receptor expression in the perivascular adipocytes and their role in hypertensive vascular injury remain unclear. The present study aimed to detect endogenous APN and its receptor expression and their protective effects against hypertensive vascular injury. APN was mainly expressed in the perivascular adipocytes, while its receptors AdipoR1 and AdipoR2 were ubiquitously expressed in the blood vessels. Angiotensin II (Ang II)-induced hypertension resulted in a significant decrease of APN and AdipoR1 and AdipoR2 in the perivascular adipocytes and vascular cells. The migration assay used demonstrated that APN attenuated Ang II-induced vascular smooth muscle cells migration and p38 phosphorylation Furthermore, the in vivo study demonstrated that APN receptor agonist AdipoRon attenuated Ang II-induced hypertensive vascular hypertrophy and fibrosis. Taken together, the present study indicated that perivascular adipocytes-derived APN attenuated hypertensive vascular injury possibly via its receptor-mediated inhibition of p38 signaling pathway.
\end{abstract}

\section{Introduction}

Adipose tissue, as a highly active endocrine organ, produces and releases a range of bioactive substances, such as adipokines, inflammatory factors and hormones $(1,2)$. Among these, adiponectin (APN) has been reported as a major anti-inflammatory mediator inhibiting the development of cardiovascular disease $(3,4)$. There is enough evidence indicating that adipocyte-derived APN can exert protective effects in different animal models such as hypertension and

Correspondence to: Dr Yanni Sun or Dr Ruimin Guo, Emergency Medicine, Putuo Hospital Affiliated to Shanghai Traditional Chinese Medicine University, 164 Lanxi Road, Shanghai 200333, P.R. China E-mail: sunstone1974@163.com

E-mail: guoruimin13597632359@126.com

Key words: adiponectin, adiponectin receptors, hypertension, vascular injury obesity (5-7). However, APN attenuates cardiac inflammation and remodeling in the classical angiotensin II (Ang II)-induced hypertension model (8). However, the potential protective role of APN on the Ang II-induced vascular remodeling has not been fully elucidated.

Adiponectin, as a fat-derived hormone, fulfills a critical role as an important messenger between adipocytes and other organs through the adiponectin receptor 1 and/or receptor 2 (AdipoR1/R2). These receptors are ubiquitously expressed in the adipose tissue, skeletal muscle and cardiovascular system $(9,10)$. Previous studies have demonstrated that APN attenuates vascular remodeling through inhibiting vascular smooth muscle cell (VSMC) proliferation and phenotype trans differentiation $(11,12)$. However, little is known about the endogenous APN and its receptor expression and function in the vasculatures of the Ang II-induced hypertensive model. In the present study, APN and AdipoR expression in vascular cells was demonstrated and the effects of APN on VSMCs migration were investigated. The distribution of these molecules in the vasculature of Ang-II induced hypertension were detected in vivo, and whether the APN receptor agonist AdipoRon could attenuate hypertension-associated vascular injury was determined.

\section{Materials and methods}

Cell culture. Vascular cells, such as human umbilical vein endothelial cells (HUVECs; American Type Culture Collection, Manassas, VA, USA) and VSMCs (PromoCell $\mathrm{GmbH}$, Heidelberg, Germany), were cultured in high glucose Dulbecco's modified Eagle's medium (DMEM) with $10 \%$ fetal bovine serum (both from Gibco; Thermo Fisher Scientific, Inc., Waltham, MA, USA) as previously described (13). Cells at 4-6 passages were used in subsequent experiments. 3T3-L1 adipocytes were differentiated from 3T3-L1 fibroblasts (American Type Culture Collection) as previously described (14). Briefly, 3T3-L1 fibroblasts were cultured to reach confluency in a 12 -well plate and were differentiated into adipocytes in the specific differentiation medium, consisting of DMEM supplemented with $10 \%$ fetal bovine serum (FBS; Gibco; Thermo Fisher Scientific, Inc.) and 3-isobutyl-1-methylxanthine $(5 \mu \mathrm{M})$, dexamethasone $(0.25 \mu \mathrm{M})$ (both from Sigma-Aldrich; Merck KGaA, Darmstadt, Germany) and insulin $(5 \mu \mathrm{g} / \mathrm{ml}$; Gibco; Thermo Fisher Scientific, Inc.) for 2 days, followed 
by treatment with DMEM supplemented with $10 \%$ FBS and insulin $(5 \mu \mathrm{g} / \mathrm{ml})$.

Animal models. Male, 8-12 week-old C57BL/6J background mice were used in the present study. To induce hypertension, mice $(\mathrm{n}=5)$ were subcutaneously infused with Ang II (Sigma-Aldrich; Merck KGaA) at a $1,500 \mathrm{ng} / \mathrm{kg} / \mathrm{min}$ dose for 14 days with ALZET mini osmotic pumps (DURECT Corporation, Cupertino, CA, USA), and in the sham group mice were treated with saline $(n=5)$. Blood pressure was measured non-invasively every day by the tail-cuff method (Softron BP-98A; Softron Co., Ltd., Tokyo, Japan). To determine the role of APN receptor in hypertensive vascular injury, Ang II-infused mice received APN receptor agonist AdipoRon ( $\mathrm{n}=5,30 \mathrm{mg} / \mathrm{kg} / \mathrm{day}$; Selleck Chemicals, Houston, TX, USA) via a gavage tube. The experiments were performed in adherence with the National Institutes of Health guidelines on the Use of Laboratory Animals and were approved by the Animal Care and Use Committee of Shanghai University of Traditional Chinese Medicine (Shanghai, China).

Histological analysis. Thoracic aortas of Ang II-induced hypertensive mice were obtained as previously described (15), and then were fixed in $4 \%$ paraformaldehyde at $4^{\circ} \mathrm{C}$ for $24 \mathrm{~h}$, embedded in paraffin, and $5 \mu \mathrm{m}$ sections were prepared for hematoxylin (15 $\mathrm{min}$ at room temperature) and eosin (5 min at room temperature) (H\&E) and picrosirius red staining (30 min at room temperature) to assess the vascular hypertrophy and fibrosis according to the manufacturer's (Wuhan Servicebio Technology Co., Ltd., Wuhan, China). Morphometric analysis was performed using Image-Pro Plus 6.0 software to assess vascular hypertrophy by measuring the medium and lumen area. Fibrotic staining was expressed as a percentage of stained areas (red) to the total areas examined. For immunofluorescence staining, paraffin sections were first deparaffinized and rehydrated in xylene $(30 \mathrm{~min})$, $100 \%$ ethanol (5 min), $90 \%$ ethanol (5 min) and $80 \%$ ethanol (5 min). Following boiling in $10 \mathrm{mM}$ sodium citrate buffer for 10 min to unmask antigens, the slides were blocked in buffer containing 5\% normal goat serum (Invitrogen; Thermo Fisher Scientific, Inc.) at room temperature for $1 \mathrm{~h}$, incubated with primary antibody at $4{ }^{\circ} \mathrm{C}$ overnight, followed by incubation with the fluorochrome-conjugated secondary antibody (1:200, for $1 \mathrm{~h}$ at room temperature; Invitrogen; Thermo Fisher Scientific, Inc.). Primary antibodies included perilin (PLIN1, 1:100, cat no. ab3526; Abcam, Cambridge, UK), APN (1:200, cat no. AF1119; R\&D Systems, Inc., Minneapolis, MN, USA), AdipoR1 (1:150, cat no. ab70362), AdipoR2 (1:150, cat no. ab77612) (both from Abcam), $\alpha$ smooth muscle actin ( $\alpha$-SMA, 1:500, cat no. A5228; Sigma-Aldrich; Merch KGaA) and p-p38 (1:100, cat no. 4511; CST Biological Reagents Co., Ltd., Shanghai, China). Images were captured by a laser scanner confocal microscopy system (LSM 710; Zeiss GmbH, Jena, Germany).

Western blot analysis. Adipocytes, VSMCs and HUVECs were lysed in a radioimmune precipitation assay buffer (0.1\% sodium dodecyl sulfate, $1.0 \%$ Triton $\mathrm{X}-100,2 \mu \mathrm{g} / \mathrm{ml}$ aprotonin and $2 \mathrm{ng} / \mathrm{ml}$ leupeptin) supplemented with protease inhibitor cocktail (Roche Diagnostics, Basel, Switzerland) on ice for $10 \mathrm{~min}$ and sonicated. Cell lysates were centrifuged $\left(12,000 \times \mathrm{g}, 15 \mathrm{~min}\right.$ at $\left.4^{\circ} \mathrm{C}\right)$ and the supernatant was preserved at $20^{\circ} \mathrm{C}$ for western blot analysis. Protein concentrations were determined using the Bradford method. Aliquots of protein $(20 \mu \mathrm{g})$ were run on a $10 \%$ sodium dodecyl sulfate-polyacrylamide gel followed by blotting onto polyvinylidene fluoride membranes by wet transfer. Membranes were blocked in blocking buffer $(5 \%$ nonfat milk in TBS buffer with $0.1 \%$ Tween-20) and were incubated with primary antibody at $4^{\circ} \mathrm{C}$ overnight. The primary antibodies included APN $(1: 2,000$, cat no. AF1119, R\&D Systems, Inc.), AdipoR1 (1:1,000, cat no. ab70362), AdipoR2 (1:1,000, cat no. ab77612) (both from Abcam), $\alpha$-SMA (1:5,000, cat no. A5228, Sigma-Aldrich; Merch KGaA), p-p38 (1:1,000, cat no. 4511), t-p38 (1:1,000, cat no. 9212) (both from CST Biological Reagents Co., Ltd.) and $\beta$-actin (1:5,000, cat no. ab8226; Abcam).The membranes were washed with TBS with Tween-20 for 3x10 min followed by incubation with the horseradish peroxidase-conjugated secondary antibodies [rabbit anti-mouse $\operatorname{IgG}(1: 5,000$, cat no. ab6728); goat anti-rabbit $\operatorname{IgG}(1: 5,000$, cat no. ab6721); rabbit anti-goat $\operatorname{IgG}(1: 5,000$, cat no. ab6741) (all from Abcam)] for $1 \mathrm{~h}$ at room temperature. Protein bands were detected by chemiluminescence (EMD Millipore, Billerica, MA, USA).

Migration assay. VSMCs migration was measured using a Transwell chamber with a polycarbonate membrane $(6.5 \mathrm{~mm}$ diameter and $8.0 \mu \mathrm{m}$ pore size; Corning Incorporated, Corning, NY, USA). Briefly, serum-starved VSMCs were trypsinized and resuspended in DMEM with $0.5 \%$ bovine serum albumin (Sigma Sigma-Aldrich; Merch KGaA). Cell suspension (100 $\mu \mathrm{l}$, $1.0 \times 10^{6}$ cells $/ \mathrm{ml}$ ) was loaded in the upper compartment of the chamber. DMEM with APN $(10 \mu \mathrm{g} / \mathrm{ml})$ were added to lower compartment and incubated at $37^{\circ} \mathrm{C}$. Following $2 \mathrm{~h}$ incubation, Ang II (100 nM) was added. After $6 \mathrm{~h}$, the cells remaining on the upper surface of the chamber were removed with a cotton swap, migrated cells on the lower surface of the chamber were fixed in $4 \%$ paraformaldehyde at room temperature for $1 \mathrm{~h}$ and stained with hematoxylin (15 $\mathrm{min}$ at room temperature) and eosin (5 min at room temperature) and three random microscopic fields (magnification, x200) per well were quantified by a fluorescence microscope (DM3000; Leica, Wetzlar, Germany).

Statistical analysis. Statistical analysis was performed using SPSS 19.0 (SPSS; IBM Corp., Armonk, NY, USA). Data are expressed as the mean \pm standard deviation. Comparisons of experimental groups were analyzed by Student's t-test (between two groups) or one-way analysis of variance, followed by the post-hoc Dunnett's test for data with more than two groups (Levene's tests for equal variance and Welch's and Brown-Forsythe's test for unequal variances). $\mathrm{P}<0.05$ was considered to indicate a statistically significant difference.

\section{Results}

Expression of APN and AdipoRs in vascular cells. APN and AdipoR expression were detected in adipocytes, VSMCs and endothelial cells (ECs) by western blot analysis. As demonstrated in Fig. 1, APN expression was highest in the adipocytes, with the expression of APN in the VSMCs and ECs less than 
A

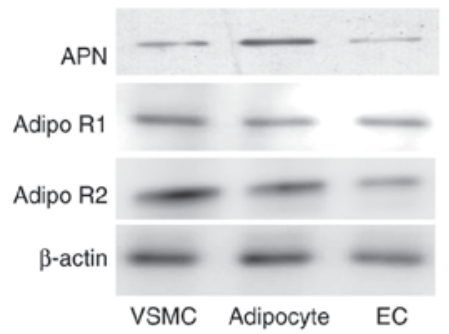

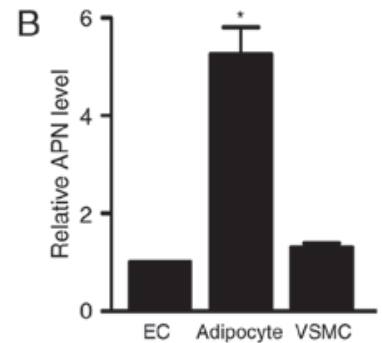
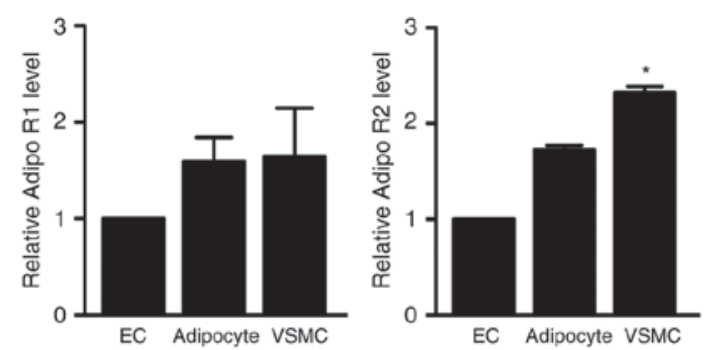

Figure 1. Representative blots of (A) APN, AdipoR1 and AdipoR2 expression in EC, VSMC and adipocytes and (B) quantification of their expression levels as assessed by western blot analysis. Data are expressed as the mean \pm standard deviation $(n=3)$. $P<0.05$ vs. EC. APN, adiponectin; AdipoR, adiponectin receptor; EC, endothelial cell; VSMC, vascular smooth muscle cell.
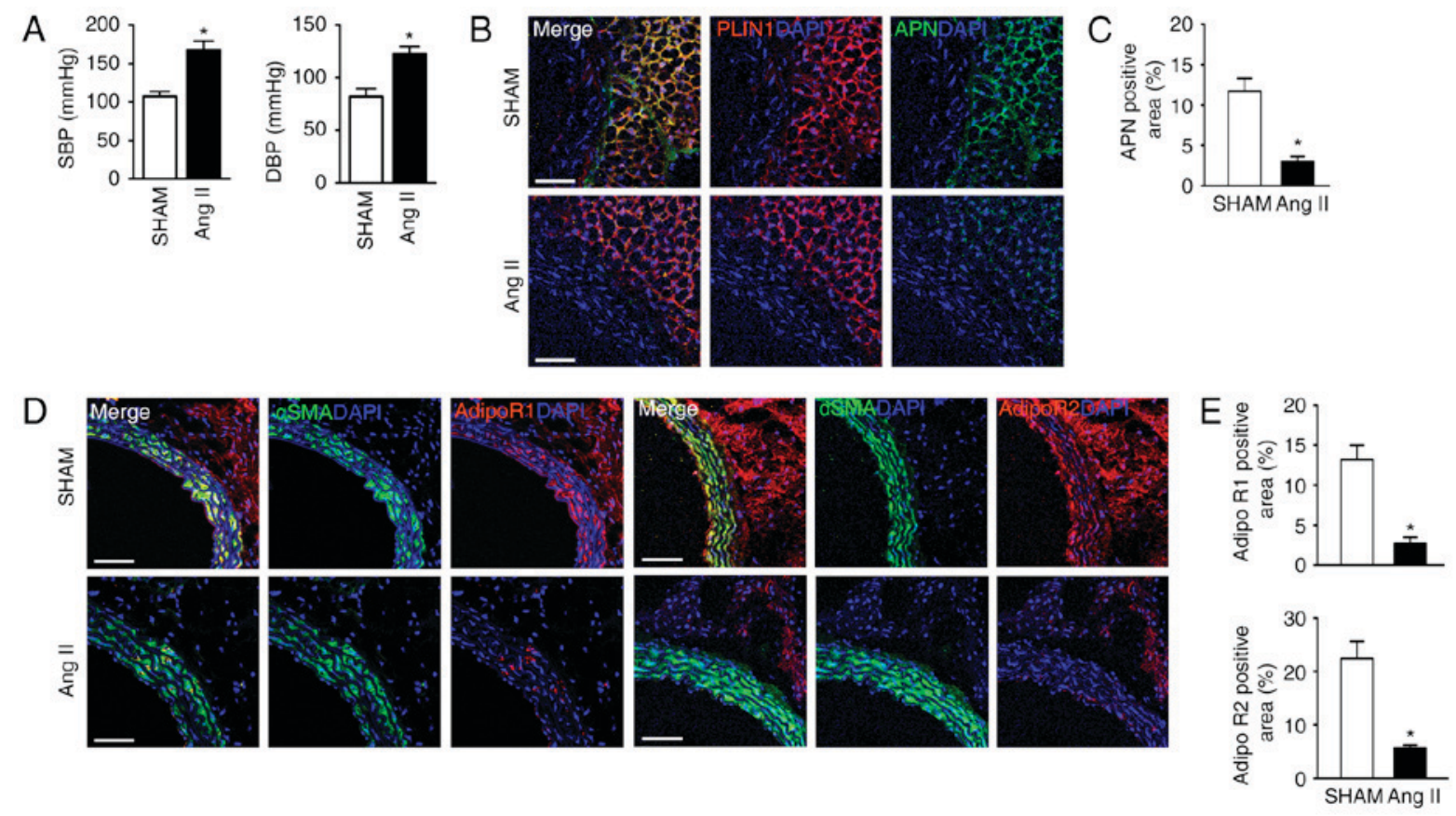

Figure 2. Expression and distribution of APN and its receptors in the vasculature. (A) Blood pressure measurements of SHAM and Ang II-infused mice. Representative immunofluorescence images of $(\mathrm{B}) \mathrm{APN}^{+}$and $\mathrm{PLIN}^{+}$cells and (C) quantification of their protein levels and representative immunofluorescence images of (D) $\alpha \mathrm{SMA}^{+}, \mathrm{AdipoR}^{+}$and AdipoR2 ${ }^{+}$cells and (E) quantification of their protein levels, in the perivascular adipocytes and VSMCs from sham and Ang II mice. DAPI counterstained for the nucleus. Scale bar, $100 \mu \mathrm{m}$. Data are expressed as the mean \pm standard deviation ( $\mathrm{n}=5$ ). " $\mathrm{P}<0.05 \mathrm{vs}$. sham. APN, adiponectin; Ang, angiotensin; VSMC, vascular smooth muscle cell; AdipoR, adiponectin receptor; SBP, systolic blood pressure; DBP, diastolic blood pressure; $\alpha$ SMA, $\alpha$ smooth muscle actin; PLIN, perilipin.

in the adipocytes. Additionally, AdipoR1 and AdipoR2 were expressed in the VSMCs, ECs and adipocytes.

Expression of APN and AdipoR in the vasculature. To detect APN and AdipoR expression in the blood vessels, immunostaining of cross-sections of thoracic aorta in Sham and Ang II-induced hypertensive mice was performed. Ang II infusion resulted in a dramatic increased blood pressure including systolic and diastolic blood pressure (Fig. 2A). In accordance with the in vitro results, APN was mainly distributed in the perivascular adipocytes (Fig. 2B and C, perilipin-positive cells), while AdipoR1 and AdipoR2 were expressed in both VSMCs ( $\alpha$ smooth muscle actin-positive cells) and adipocytes (Fig. 2D and E). Ang II-induced hypertension resulted in decreased APN and AdipoR expression in the vasculature.

Effects of Ang II on APN and AdipoR expression. To detect the direct effects of Ang II on APN, APN levels in the 3T3-L1 adipocytes pretreated with Ang II were measured. The result demonstrated that APN expression was decreased in a time-dependent manner following Ang II treatment (Fig. 3A). In addition, it was also illustrated that AdipoR1 and AdipoR2 expression was decreased in a time-dependent manner following Ang II treatment in the VSMCs (Fig. 3B).

APN attenuates VSMCs migration. Vascular active substance-induced VSMCs migration serves a pivotal role in hypertension-associated vascular remodeling (16). Whether APN affects Ang II-induced VSMCs migration was examined by utilizing the Transwell chamber assay. As depicted in Fig. 4A, APN $(10 \mu \mathrm{g} / \mathrm{ml})$ significantly attenuated Ang II-induced VSMCs migration. The p38 signaling pathway is known to be involved in the regulation of VSMC migration (17) In the present study, the phosphorylation of p38 mitogen-activated protein kinase (MAPK) was reduced following APN treatment in Ang II-stimulated VSMCs (Fig. 4B). 
A
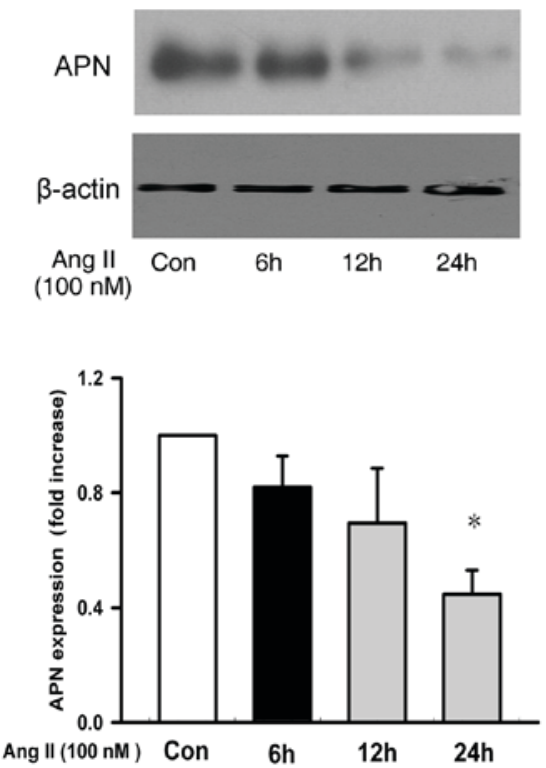

B

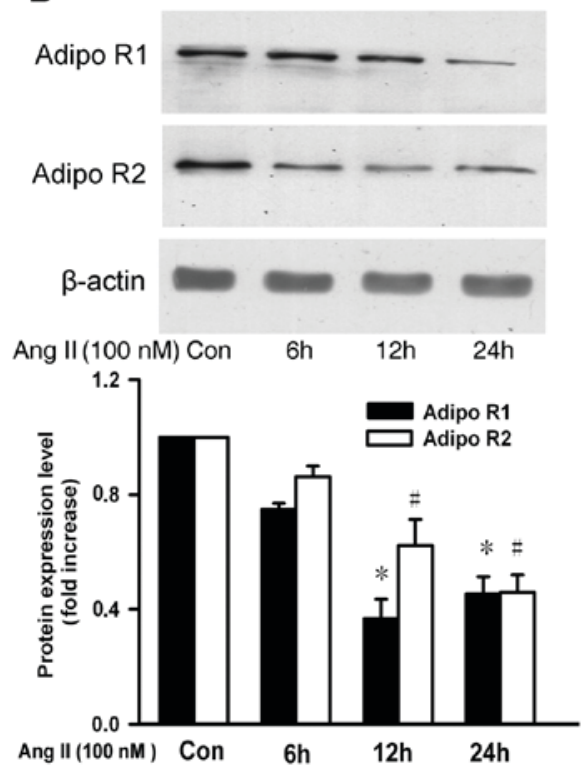

Figure 3. APN and its receptors expression levels following Ang II treatment. Representative blots of (A) APN in 3T3-L1 adipocytes and (B) AdipoR1/2 in vascular smooth muscle cells following Ang II treatment. Data are expressed as the mean \pm standard deviation $\left(\mathrm{n}=3\right.$ ). ${ }^{*} \mathrm{P}<0.05$, ${ }^{\#} \mathrm{P}<0.05 \mathrm{vs}$. Con. APN, adiponectin; Ang II, angiotensin II; AdipoR, adiponectin receptor.

A
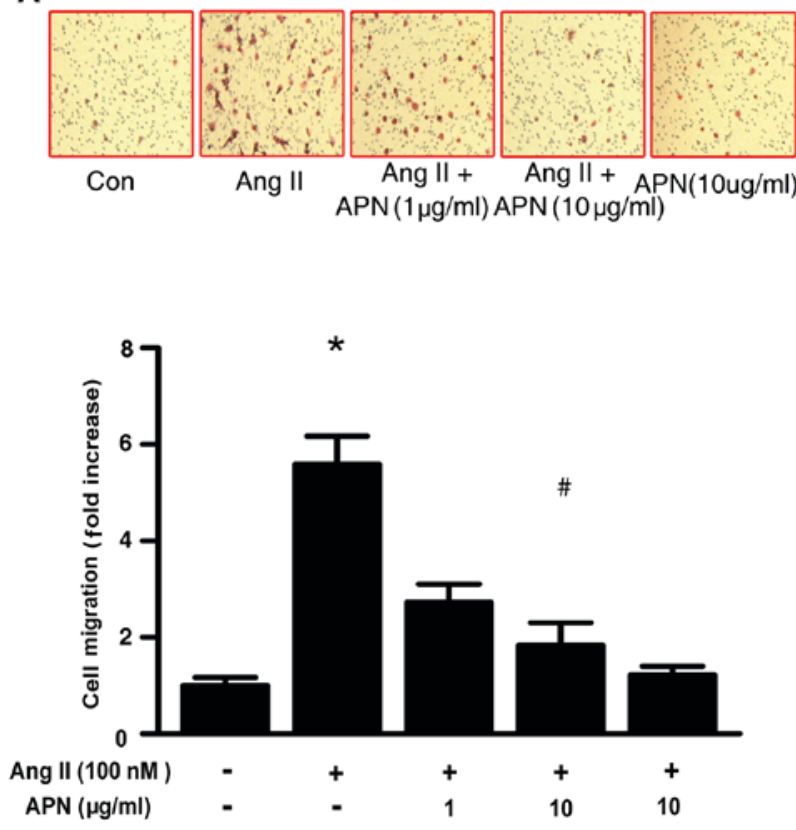

B
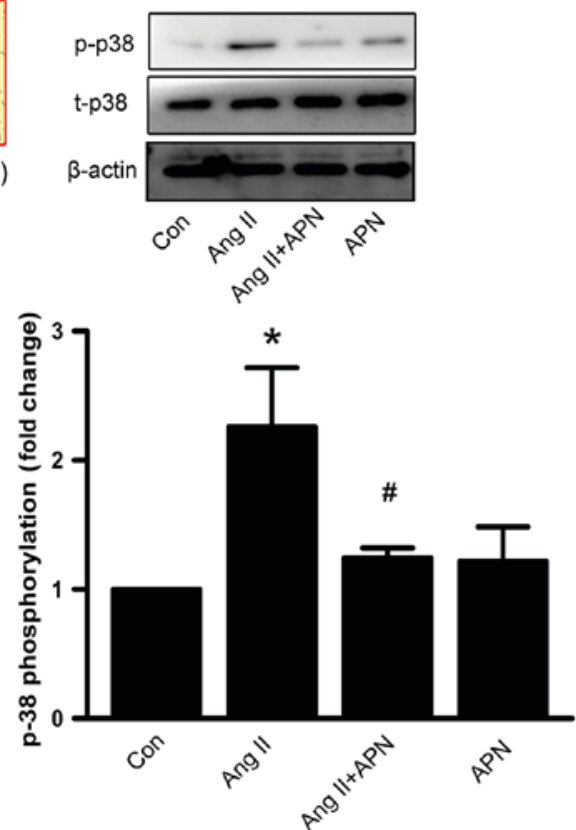

Figure 4. APN inhibits vascular smooth muscle cell migration and p38 phosphorylation stimulated by Ang II. Representative image of (A) migrated cells stained with hematoxylin and eosin, as assessed by the migration assay and its quantification. (B) Representative blots of the p-p38 and t-p38 and quantification of its protein levels. Scale bar, $100 \mu \mathrm{m}$. Data are expressed as the mean \pm standard deviation $(\mathrm{n}=3)$. ${ }^{*} \mathrm{P}<0.05$ vs. Con, ${ }^{\sharp} \mathrm{P}<0.05$ vs. Ang II. APN, adiponectin; Ang II, angiotensin II; p, phosphorylated; t, total.

AdipoRon attenuates hypertensive vascular remodeling. AdipoRon is an orally active APN receptor activator, which potentially exerts protective effects against cardiac remodeling $(18,19)$. In order to detect the effects of AdipoRon in hypertension-associated vascular remodeling, the Ang II-infused mice were orally treated with AdipoRon $(30 \mathrm{mg} / \mathrm{kg} /$ day $)$. As demonstrated in Fig. 5A, AdipoRon treatment resulted in decreased p38 phosphorylation. In addition,
AdipoRon attenuated Ang II-induced perivascular collagen deposition and vascular hypertrophy as detected by Sirious Red and H\&E stainings (Fig. 5B and C, respectively).

\section{Discussion}

In the present study, the distribution of APN and AdipoR in the vasculature was detected and it was demonstrated that APN 

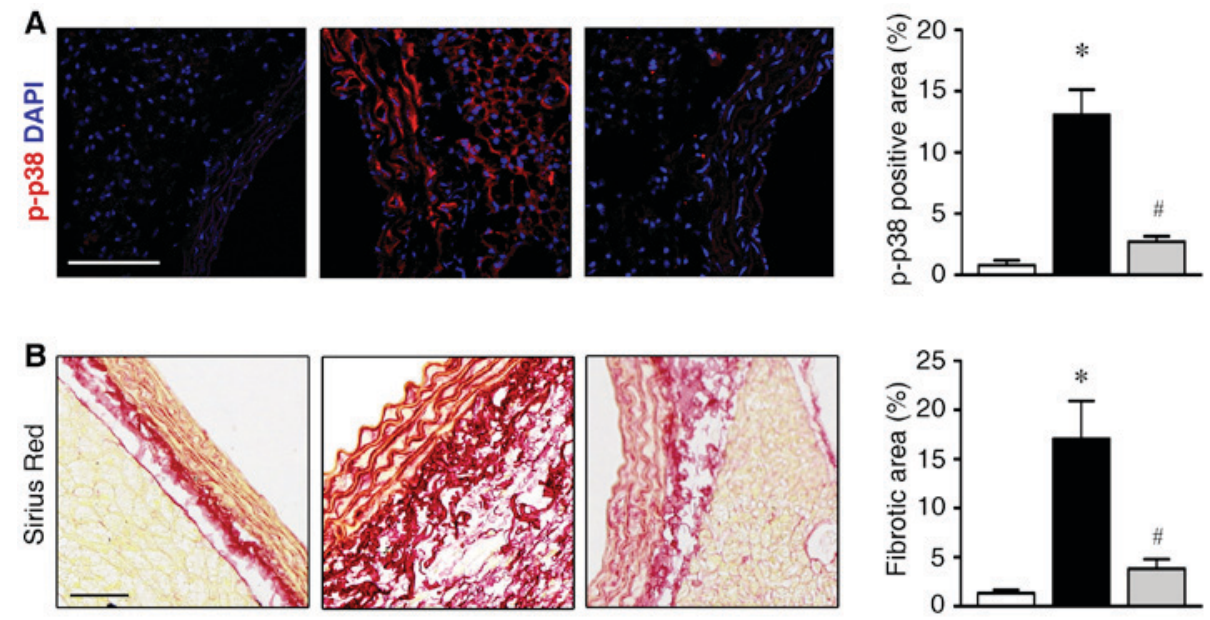

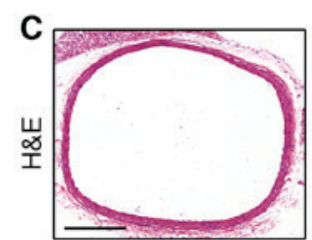

SHAM

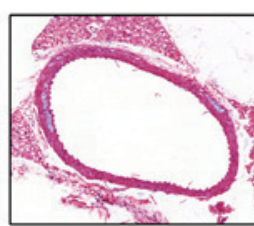

Ang II

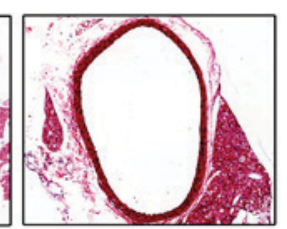

Ang II + AdipoRon

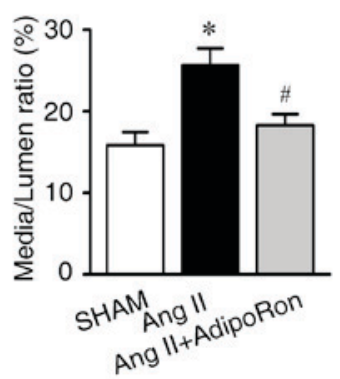

Figure 5. AdipoRon attenuates Ang II-induced vascular injury. (A) Immunofluorescence analysis of p-p38 and quantification of its protein expression levels, in Sham, Ang II and Ang II+ AdipoRon-treated mice (scale bar, $100 \mu \mathrm{m}$ ). Representative images of (B) Sirius Red (scale bar, $200 \mu \mathrm{m})$ and (C) hematoxylin and eosin staining (scale bar, $50 \mu \mathrm{M}$ ) of aorta cross sections from sham, Ang II and Ang II+ AdipoRon-treated mice. Data are expressed as the mean \pm standard deviation ( $\mathrm{n}=5)$. ${ }^{*} \mathrm{P}<0.05$ vs. sham, ${ }^{\#} \mathrm{P}<0.05$ vs. Ang II. Ang II, angiotensin; p-p38, phosphorylated-p38.

was predominantly expressed in the perivascular adipocytes, while AdipoR1 and R2 were ubiquitously expressed in the blood vessels. APN attenuated Ang II-induced VSMCs migration and p38 phosphorylation. Furthermore, APN receptor agonist, AdipoRon, appeared to attenuate Ang II-induced hypertensive vascular hypertrophy and fibrosis.

Perivascular adipocytes are an important source of adipokines and their role in regulating vascular function is well established (20-22). APN, an abundant adipocyte-derived factor with a variety of biological functions, was first described in 1995 (23). Over the past two decades, numerous studies have elucidated its physiological functions, which may improve insulin sensitivity in insulin target tissues, modulate inflammatory responses, and serve a crucial role in the regulation of energy metabolism $(24,25)$. In the cardiovascular system, APN ameliorates Ang II-induced cardiac remodeling by attenuating inflammation, it inhibits connective tissue growth factor-induced VSMCs proliferation, and promotes endothelial nitric oxide synthase expression and nitric oxide release from endothelial cells $(24,26)$. Previous studies have demonstrated that hypertensive mice express decreased APN plasma levels compared with the controls (27). To the best of our knowledge, the present study was the first to demonstrate that hypertension induced APN downregulation in local perivascular adipocytes. VSMC dysfunction, including migration, proliferation and phenotype transdifferentiation, has an important role in hypertension-induced vascular remodeling. VSMC migration is associated with vascular remodeling when exposed to high stress, such as hypertension. It has been demonstrated that
Ang II significantly increased the VSMC migration (28). APN receptors, AdipoR1 and AdipoR2, were originally identified by screening a skeletal muscle expression library for cDNAs that encoded proteins that to the globular domain of APN (29). Both receptors contain seven transmembrane domains and belong to the progestin and adipoQ receptor family, which has the opposite transmembrane topology to the G-protein coupled receptors, and have the N-terminus in the cytoplasm and the C-terminus facing the extracellular space. Although ubiquitously expressed, AdipoR1 is most abundant in skeletal muscle whereas AdipoR2 is mainly restricted to the liver $(30,31)$. In the present study, ubiquitous expression of AdipoR1 and AdipoR2 in the vasculature was demonstrated, including ECs, VSMCs and perivascular adipocytes. In an obesity and diabetic mouse model, AdipoR1 expression was reduced in the target tissue $(32,33)$. However, in a rat model of chronic kidney disease, AdipoR1 and AdipoR2 expression was significantly increased in renal cells (34). In the present study, AdipoR1 and AdipoR2 expression was decreased in the cultured VSMCs treated with Ang II. In addition, Ang II-induced hypertension resulted in a significant decrease of AdipoR1 and AdipoR2 expression in the perivascular adipocytes and VSMCs in vivo. AdipoR1 or AdipoR2 mediate the effects of APN by regulating multiple signaling pathways, including the peroxisome proliferator-activated receptor- $\alpha$, the AMP-activated protein kinase, and the p38 MAPK $(12,35,36)$. Previous studies have demonstrated that APN could attenuate lipid deposition and apoptosis through activating p38 MAPK pathway $(37,38)$. In the present study, it was demonstrated that APN alone could induce 
p38 phosphorylation, which is in accordance with previous studies $(39,40)$. Furthermore, Ang II promoted p38 phosphorylation. It could be speculated that the APN/p38 axis may have an inhibitory feedback effect on $\mathrm{p} 38$ phosphorylation, with p38 phosphorylation level is decreased following Ang II treatment. APN receptor agonist, AdipoRon, inhibited p38 phosphorylation in the vasculature of Ang II-induced hypertensive mice.

In conclusion, the present study demonstrated that Ang II-induced hypertensive mice had decreased expression of APN and AdipoR in the perivascular adipocytes and VSMCs. APN attenuated Ang II-induced VSMCs migration and p38 phosphorylation. APN receptor agonist AdipoRon attenuated Ang II-induced hypertensive vascular remodeling. These results suggest that APN and APN receptors may be potential therapeutic targets for inhibiting hypertensive vascular injury.

\section{Acknowledgements}

The present study was supported by the Putuo Hospital Level Subject (AASI index in old hypertension patients complicated with metabolism diseases; grant no. PT65487).

\section{References}

1. Thalmann S and Meier CA: Local adipose tissue depots as cardiovascular risk factors. Cardiovasc Res 75: 690-701, 2007.

2. Molica F, Morel S, Kwak BR, Rohner-Jeanrenaud F and Steffens S: Adipokines at the crossroad between obesity and cardiovascular disease. Thromb Haemost 113: 553-566, 2015.

3. Li FY, Cheng KK, Lam KS, Vanhoutte PM and Xu A: Cross-talk between adipose tissue and vasculature: Role of adiponectin. Acta Physiol (Oxf) 203: 167-180, 2011.

4. Cai X, Li X, Li L, Huang XZ, Liu YS, Chen L, Zhang K, Wang L, $\mathrm{Li} \mathrm{X}$, Song J, et al: Adiponectin reduces carotid atherosclerotic plaque formation in ApoE-/-mice: Roles of oxidative and nitrosative stress and inducible nitric oxide synthase. Mol Med Rep 11: 1715-1721, 2015.

5. Withers SB, Bussey CE, Saxton SN, Melrose HM, Watkins AE and Heagerty AM: Mechanisms of adiponectin-associated perivascular function in vascular disease. Arterioscler Thromb Vasc Biol 34: 1637-1642, 2014.

6. Liu Y, Palanivel R, Rai E, Park M, Gabor TV, Scheid MP, Xu A and Sweeney G: Adiponectin stimulates autophagy and reduces oxidative stress to enhance insulin sensitivity during high-fat diet feeding in mice. Diabetes 64: 36-48, 2015.

7. Li C, Wang Z, Wang C, Ma Q and Zhao Y: Perivascular adipose tissue-derived adiponectin inhibits collar-induced carotid atherosclerosis by promoting macrophage autophagy. PLoS One 10: e0124031, 2015 .

8. Qi GM, Jia LX, Li YL, Li HH and Du J: Adiponectin suppresses angiotensin II-induced inflammation and cardiac fibrosis through activation of macrophage autophagy. Endocrinology 155: 2254-2265, 2014.

9. Yamauchi T, Kamon J, Ito Y, Tsuchida A, Yokomizo T, Kita S, Sugiyama T, Miyagishi M, Hara K, Tsunoda M, et al: Cloning of adiponectin receptors that mediate antidiabetic metabolic effects. Nature 423: 762-769, 2003.

10. Wang ZV and Scherer PE: Adiponectin, the past two decades. J Mol Cell Biol 8: 93-100, 2016.

11. Zhan JK, Wang YJ, Wang Y, Tang ZY, Tan P, Huang W and Liu YS: Adiponectin attenuates the osteoblastic differentiation of vascular smooth muscle cells through the AMPK/mTOR pathway. Exp Cell Res 323: 352-358, 2014.

12. Zhang W, Shu C, Li Q, Li M and Li X: Adiponectin affects vascular smooth muscle cell proliferation and apoptosis through modulation of the mitofusin-2-mediated Ras-Raf-Erk1/2 signaling pathway. Mol Med Rep 12: 4703-4707, 2015.

13. Alexis JD, Wang N, Che W, Lerner-Marmarosh N, Sahni A, Korshunov VA, Zou Y, Ding B, Yan C, Berk BC and Abe J: Bcr kinase activation by angiotensin II inhibits peroxisome-proliferator-activated receptor gamma transcriptional activity in vascular smooth muscle cells. Circ Res 104: 69-78, 2009.
14. Yang Z, Hong LK, Follett J, Wabitsch M, Hamilton NA, Collins BM, Bugarcic A and Teasdale RD: Functional characterization of retromer in GLUT4 storage vesicle formation and adipocyte differentiation. Faseb J 30: 1037-1050, 2016.

15. Zhao Y, Zhang C, Wei X, Li P, Cui Y, Qin Y, Wei X, Jin M, Kohama K and Gao Y: Heat shock protein 60 stimulates the migration of vascular smooth muscle cells via Toll-like receptor 4 and ERK MAPK activation. Sci Rep 5: 15352, 2015.

16. Montezano AC, Nguyen Dinh Cat A, Rios FJ and Touyz RM: Angiotensin II and vascular injury. Curr Hypertens Rep 16: 431, 2014.

17. Yu L, Huang X, Huang K, Gui C, Huang Q and Wei B: Ligustrazine attenuates the platelet-derived growth factor-BB-induced proliferation and migration of vascular smooth muscle cells by interrupting extracellular signal-regulated kinase and P38 mitogen-activated protein kinase pathways. Mol Med Rep 12: 705-711, 2015.

18. Okada-Iwabu M, Yamauchi T, Iwabu M, Honma T, Hamagami K, Matsuda K, Yamaguchi M, Tanabe H, Kimura-Someya T, Shirouzu M, et al: A small-molecule AdipoR agonist for type 2 diabetes and short life in obesity. Nature 503: 493-499, 2013.

19. Fisman EZ and Tenenbaum A: Adiponectin: A manifold therapeutic target for metabolic syndrome, diabetes and coronary disease? Cardiovasc Diabetol 13: 103, 2014.

20. Takaoka M, Nagata D, Kihara S, Shimomura I, Kimura Y, Tabata Y, Saito Y, Nagai R and Sata M: Periadventitial adipose tissue plays a critical role in vascular remodeling. Circ Res 105: 906-911, 2009.

21. Sheng LJ, Ruan CC, Ma Y, Chen DR, Kong LR, Zhu DL and Gao PJ: Beta3 adrenergic receptor is involved in vascular injury in deoxycorticosterone acetate-salt hypertensive mice. FEBS lett 590: 769-778, 2016.

22. Takaoka M, Suzuki H, Shioda S, Sekikawa K, Saito Y, Nagai R and Sata M: Endovascular injury induces rapid phenotypic changes in perivascular adipose tissue. Arterioscler Thromb Vasc Biol 30: 1576-1582, 2010.

23. Scherer PE, Williams S, Fogliano M, Baldini G and Lodish HF: A novel serum protein similar to Clq, produced exclusively in adipocytes. J Biol Chem 270: 26746-26749, 1995.

24. Lee $S$ and Kwak HB: Role of adiponectin in metabolic and cardiovascular disease. J Exerc Rehabil 10: 54-59, 2014.

25. Chakraborti CK: Role of adiponectin and some other factors linking type 2 diabetes mellitus and obesity. World J Diabetes 6: 1296-1308, 2015

26. Freitas Lima LC, Braga VA, do Socorro de Franca Silva M, Cruz JC, Sousa Santos SH, de Oliveira Monteiro MM and Balarini CM: Adipokines, diabetes and atherosclerosis: An inflammatory association. Front Physiol 6: 304, 2015.

27. Ndisang JF and Jadhav A: Heme arginate therapy enhanced adiponectin and atrial natriuretic peptide, but abated endothelin-1 with attenuation of kidney histopathological lesions in mineralocorticoid-induced hypertension. J Pharmacol Exp Ther 334: 87-98, 2010.

28. Zhang F, Ren X, Zhao M, Zhou B and Han Y: Angiotensin-(1-7) abrogates angiotensin II-induced proliferation, migration and inflammation in VSMCs through inactivation of ROS-mediated PI3 K/Akt and MAPK/ERK signaling pathways. Sci Rep 6: $34621,2016$.

29. Kadowaki $\mathrm{T}$ and Yamauchi $\mathrm{T}$ : Adiponectin and adiponectin receptors. Endocr Rev 26: 439-451, 2005.

30. Yamauchi $\mathrm{T}$ and Kadowaki T: Adiponectin receptor as a key player in healthy longevity and obesity-related diseases. Cell Metab 17: 185-196, 2013.

31. Lustig Y, Hemi R and Kanety H: Regulation and function of adiponectin receptors in skeletal muscle. Vitam Horm 90: 95-123, 2012.

32. Wade TE, Mathur A, Lu D, Swartz-Basile DA, Pitt HA and Zyromski NJ: Adiponectin receptor-1 expression is decreased in the pancreas of obese mice. J Surg Res 154: 78-84, 2009.

33. Lin T, Qiu Y, Liu Y, Mohan R, Li Q and Lei B: Expression of adiponectin and its receptors in type 1 diabetes mellitus in human and mouse retinas. Mol Vis 19: 1769-1778, 2013.

34. Yu Y, Bao BJ, Fan YP, Shi L and Li SQ: Changes of adiponectin and its receptors in rats following chronic renal failure. Ren Fail 36: 92-97, 2014.

35. Thundyil J, Pavlovski D, Sobey CG and Arumugam TV: Adiponectin receptor signalling in the brain. Br J Pharmacol 165: 313-327, 2012.

36. Almabrouk TA, Ewart MA, Salt IP and Kennedy S: Perivascular fat, AMP-activated protein kinase and vascular diseases. Br J Pharmacol 171: 595-617, 2014. 
37. Yan J, Gan L, Qi R and Sun C: Adiponectin decreases lipids deposition by 38 MAPK/ATF2 signaling pathway in muscle of broilers. Mol Biol Rep 40: 7017-7025, 2013

38. Wang Y, Zhang J, Zhang L, Gao P and Wu X: Adiponectin attenuates high glucose-induced apoptosis through the AMPK/p38 MAPK signaling pathway in NRK-52E cells. PLoS One 12: e0178215, 2017.

39. $\mathrm{Pu} \mathrm{Y}$, Wang M, Hong $\mathrm{Y}, \mathrm{Wu} \mathrm{Y}$ and Tang Z: Adiponectin promotes human jaw bone marrow mesenchymal stem cell chemotaxis via CXCL1 and CXCL8. J Cell Mol Med 21: 1411-1419, 2017.
40. Huang CY, Chang AC, Chen HT, Wang SW, Lo YS and Tang CH: Adiponectin promotes VEGF-C-dependent lymphangiogenesis by inhibiting miR-27b through a CaMKII/AMPK/p38 signaling pathway in human chondrosarcoma cells. Clin Sci (Lond) 130: $1523-1533,2016$

(i)(9) This work is licensed under a Creative Commons EY NG ND Attribution-NonCommercial-NoDerivatives 4.0 International (CC BY-NC-ND 4.0) License. 